\title{
Publisher Correction: Observation-based early-warning signals for a collapse of the Atlantic Meridional Overturning Circulation
}

Niklas Boers (D)

Correction to: Nature Climate Change https://doi.org/10.1038/s41558-021-01097-4, published online 5 August 2021.

In the version of this Article initially published, an error appeared in the Data Availability statement in the url "http://www.pik-potsdam. de/caesar/AMOC_slowdown/”. The site link has been corrected to read: “http://www.pik-potsdam.de/ caesar/AMOC_slowdown/”.

The original Article has been corrected in the online version of the paper.

Published online: 17 September 2021

https://doi.org/10.1038/s41558-021-01184-6

(c) The Author(s), under exclusive licence to Springer Nature Limited 2021 